\title{
DISCRETE AIRY BEAM PROPAGATION IN DEEP OCEANS
}

\author{
Marcelo Diniz Santa Marinha ${ }^{1}$, Danian S. Oliveira ${ }^{1}$, Paulo Eduardo Miranda Cunha² \\ and Luiz Gallisa Guimarães ${ }^{1}$
}

ABSTRACT. In this work, based on the theory of modal propagation of acoustic energy in deep oceans and using the analogy between acoustics and quantum mechanics, we show that it is possible the existence of almost dispersionless Airy beams propagation at great distances with simultaneous wave packet shape preservation.

Keywords: normal modes, wave propagation, ocean acoustics, Airy beam.

RESUMO. Neste trabalho, baseado na teoria de propagação da energia acústica por modos em oceanos profundos, mostraremos que é possível a existência de feixes de Airy que se propagam em grandes distâncias, com pouca dispersão da energia acústica e preservação de sua forma.

Palavras-chave: modos normais, propagação de ondas, acústica oceanográfica, feixe de Airy.

\footnotetext{
${ }^{1}$ Universidade Federal do Rio de Janeiro (UFRJ), Instituto Alberto Luiz Coimbra de Pós-Graduacão e Pesquisa de Engenharia - Engenharia Naval e Oceânica, Av. Athos Silveira, 149 CT - Bloco C - Sala 203, Cidade Universitária - Ilha do Fundão, 21941-972, Rio de Janeiro, RJ, Brazil - E-mails: marcelodsmarinha@gmail.com, danian.oliveira@gmail.com, Lgg@oceanica.ufrj.br

${ }^{2}$ Petrobras, Centro de Pesquisas e Desenvolvimento Leopoldo Américo Miguez de Mello (Cenpes), Av. Horácio Macedo, 950, 21941-915, Rio de Janeiro, RJ, Brazil E-mail: pemcunha@gmail.com
} 


\section{INTRODUCTION AND MOTIVATIONS}

In the late 70s, Berry \& Balazs (1979) published a pioneering work on the foundations of quantum mechanics calling attention to the important fact that, even for a free particle type problem, a wave packet (representing these particles) in the form of Airy beams are accelerated, and even more, such beams minimize diffractive effects throughout their propagation (Durnin et al., 1987; Siviloglou \& Christodoulides, 2007).

In other words, in addition to preserving the transverse shape of the pulses, the Airy beam propagates its main amplitude along a parabolic path. Associated with this movement, the beam is said to be self-accelerating.

Therefore, motivated by similar results, both theoretical (Berry, 2005) and experimental, in areas involving wave propagation, such as optics (Salandrino \& Christodoulides, 2011), underwater acoustics and ocean waves (Fu et al., 2017), we have developed a theoretical model that accurately describes the propagation of Airy beams in an acoustic waveguide represented by a deep and unlimited ocean.

For this purpose, we have created an acoustic propagation algorithm which, simultaneously, takes into account certain characteristics of ray tracing (Popov, 2002; Cerveny, 2005; Bleistein, 2008), together with the theory of normal modes in the ocean (Porter \& Bucker, 1987; Jensen, 1994).

In the next section we will present a brief review on the semi-classical WKB theory of acoustic propagation by normal modes and ray tracing in deep oceans. We will show that under certain conditions, these theories provide equivalent results and subsidies for the development of a discrete Airy beam propagation model. Finally, in the last section, we will summarize our results and discuss possible outcomes.

\section{METHODOLOGY: FUNDAMENTALS OF THE PROPAGATION OF GENERALIZED AIRY BEAMS}

\section{WKB analysis for modal propagation in the Munk canonical acoustic guide}

For harmonic acoustic displacement fields $\Phi$, with a time variation $\exp (-i \omega t)$, propagating in an ocean with density $\rho(\vec{r})$ and sound velocity profile $c(\vec{r})$, it is assumed that the acoustic pressure $P$, in the frequency domain, behaves as $P=\rho \omega^{2} \Phi$, where $\omega=2 \pi f$ is the angular frequency Jensen (1994).

Within this formalism and for cases where the density $\rho$ is constant or varies smoothly throughout the water layer, the acoustic displacement field $\Phi$, generated by point sources, are the propagating solutions of Helmholtz wave equation (Jensen, 1994)

$$
\left(\nabla^{2}+\left[\frac{\omega}{c(\vec{r})}\right]^{2}\right) \Phi=A \delta\left(\vec{r}-\vec{r}_{s}\right),
$$

where, $A$ is the amplitude of the signal emitted by a source positioned at $\vec{r}=\vec{r}_{s}$. In deep oceans, due to the azimuthal symmetry of the problem, we will adopt the polar cylindrical coordinate system, such that the ocean depth is represented by the $z$-axis, while $r$ is the radial distance to this axis.

In deep water, since the sound velocity variability in depth is greater than in distance, we can assume $c(\vec{r}) \approx c(z)$ where $r$ can vary by some tens of kilometers and $z$ few thousand meters. Under these conditions, the general solution of Eq. (1) can be described by the following Hankel transform (Jensen, 1994)

$$
\Phi\left(r, z, z_{s}\right)=\frac{1}{2} \int_{-\infty}^{+\infty} d k_{r} \Psi\left(z, z_{s}\right) k_{r} H_{0}^{1}\left(k_{r} r\right),
$$

where $H_{0}^{1}$ is the first kind and zero order Hankel function. This solution is compatible with the Sommerfeld radiation condition in cylindrical coordinates. Moreover, the Green function $\Psi$ satisfies the differential equation Jensen (1994)

$$
\frac{\partial^{2} \Psi}{\partial z^{2}}+\left[\left(\frac{\omega}{z} c(z)\right)^{2}-k_{r}^{2}\right] \Psi=A \delta\left(z-z_{s}\right) .
$$

Unfortunately in the case of Munk's canonical profile (Fig. 1), described by Eq. (4) related to hydrodynamic acoustical parameters $L$ and $\varepsilon$,

$$
c(z) \equiv c_{\min }\left(1+\varepsilon\left[2 \frac{\left(z-z_{\min }\right)}{L}-1+\exp \left(-2 \frac{\left(z-z_{\min }\right)}{L}\right)\right]\right),
$$

it is not possible to solve explicitly the differential equation (Eq. 3).

However we can gain some physical insights about the problem, initially restricting our analysis at depths below the mixed layer (Fig. 1). It means that, in a first approach, we will not consider the reflection of the propagating waves on the surface of the ocean and we will use approximate methods, as the WKB theory.

For example, within this approximation, it is known that the propagating modes are associated with discrete values of the radial wave number, $k_{r} \rightarrow k_{r}^{[n]}$, such that $k_{r}^{[n]}$ must satisfy the Bohr-Sommerfeld quantization criterion (Jensen, 1994)

$$
\int_{z_{\mathrm{A}}}^{z_{B}} d z k_{z}^{[n]}(z)=\left(n+\frac{1}{2}\right) \pi ; n=0,1, \ldots, n_{\max },
$$


where, for any of the $n$ 's, among the $n_{\max }+1$ possible positive integers, we define $k_{z}^{[n]}$ as the discrete vertical wave number

$$
k_{z}^{[n]}(z) \equiv \sqrt{\left(\frac{\omega}{c(z)}\right)^{2}-k_{r}^{[n] 2}},
$$

which, together with Eq. (5), show us that the integration limits $z_{A}$ and $z_{B}$ are the classical turning points, and they are the real solutions of

$$
k_{z}^{[n]}\left(z_{A}\right)=0=k_{z}^{[n]}\left(z_{B}\right)
$$

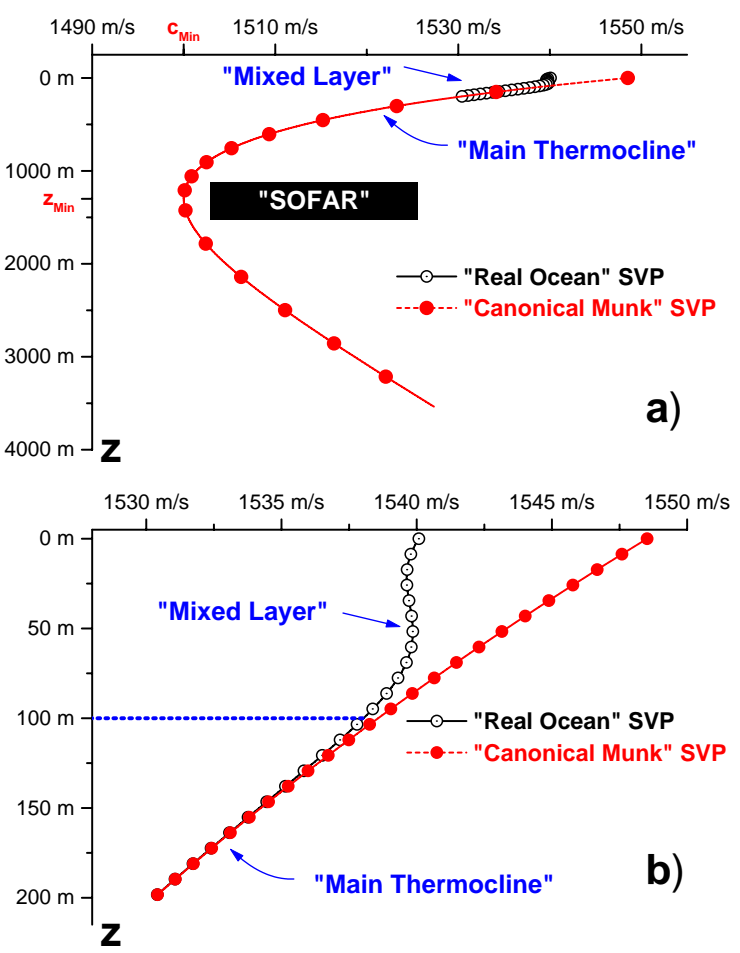

Figure 1 - Comparison between typical sound velocity profiles and the theoretical model of Munk (Eq. 4), as a function of depth, for deep waters. In a) we see that, in the deeper layers, the behavior of both profiles resemble both the thermocline and the SOFAR. However, in b), we see that the Munk profile (Eq. 4) does not satisfactorily describe the velocity behavior of sound in shallow waters relative to the interior of the mixed layer.

It is also observed, from Eq. (6), that $k_{z}^{[n]}$ reaches its maximum value, $k_{z}^{[\operatorname{Max}, n]}$, inside the SOFAR (Sound Fixing and Ranging) channel when $z=z_{\text {min }}$ (Jensen, 1994). In Jensen (1994), we also find typical values referring to the minimum depths and sound velocities, respectively $z_{\min }=1300 \mathrm{~m}$ and $c_{\text {min }}=1500 \mathrm{~m} / \mathrm{s}$. Associated with these parameters we can define the cutoff frequency of the Munk waveguide as

$$
f_{c} \equiv \frac{c_{\min }}{2 z_{\min }},
$$

which is the smallest frequency below which there can be no propagating waves inside the guide. Throughout this work, we have adopted a frequency of $50 \mathrm{~Hz}$, which is about eighty times greater than the cutoff frequency of a typical Munk guide. In addition, we will explicitly adopt the following values for deep water, $L=z_{\min }=1300 \mathrm{~m}$ and $\varepsilon=0.00737$, referring to Eq. (4), wich characterize the parameters of Munk profile (Fig. 1).

In the early 80's, Chapman \& Ellis (1983), using the WKB formalism, showed that it is possible to relate the ray trajectories, which have passed at least once through the return point, to a specific set of adjacent normal modes. An analogy was made between the time, $\tau_{\Gamma}$, that a wave travels through a trajectory of length $\Gamma$,

$$
\tau_{\Gamma} \equiv \int_{\Gamma} \frac{d \ell}{c},
$$

and the time scale, $\tau_{n}$, of two adjacent modes, $n$ and $n+1$, inside a waveguide, that traveled radially through half modal interference length $L_{n}$, expressed by

$$
\tau_{n} \equiv \frac{L_{n}}{c_{g}^{[n]}} .
$$

In situations of low dispersion of the acoustic wave field (Chapman \& Ellis, 1983), we have $\tau_{\Gamma} \approx \tau_{n}$ (Fig. 2).

According to Jensen (1994), $L_{n}$, in Eq. (10), satisfies

$$
L_{n} \equiv \frac{\pi}{k_{r}^{[n]}-k_{r}^{[n+1]}},
$$

and the modal group velocity, $c_{g}^{[n]}$, is denoted by

$$
c_{g}^{[n]}=\frac{\partial \omega}{\partial k_{r}^{[n]}} .
$$

Similarly, as seen in Jensen (1994) and Chapman \& Ellis (1983), for a given frequency $\omega$, the modal phase velocity, $c_{p}^{[n]}$, is defined by

$$
c_{p}^{[n]} \equiv \frac{\omega}{k_{r}^{[n]}} .
$$

In addition, for a given mode $n$, from Eqs. (6), (7) and (13), we can see that the sound velocity equals the modal phase velocity at the turning points $z_{A} \mapsto z_{A}^{[n]}$ and $z_{B} \mapsto z_{B}^{[n]}$,

$$
c\left(z_{A}\right)=c_{p}^{[n]}=c\left(z_{B}\right) .
$$




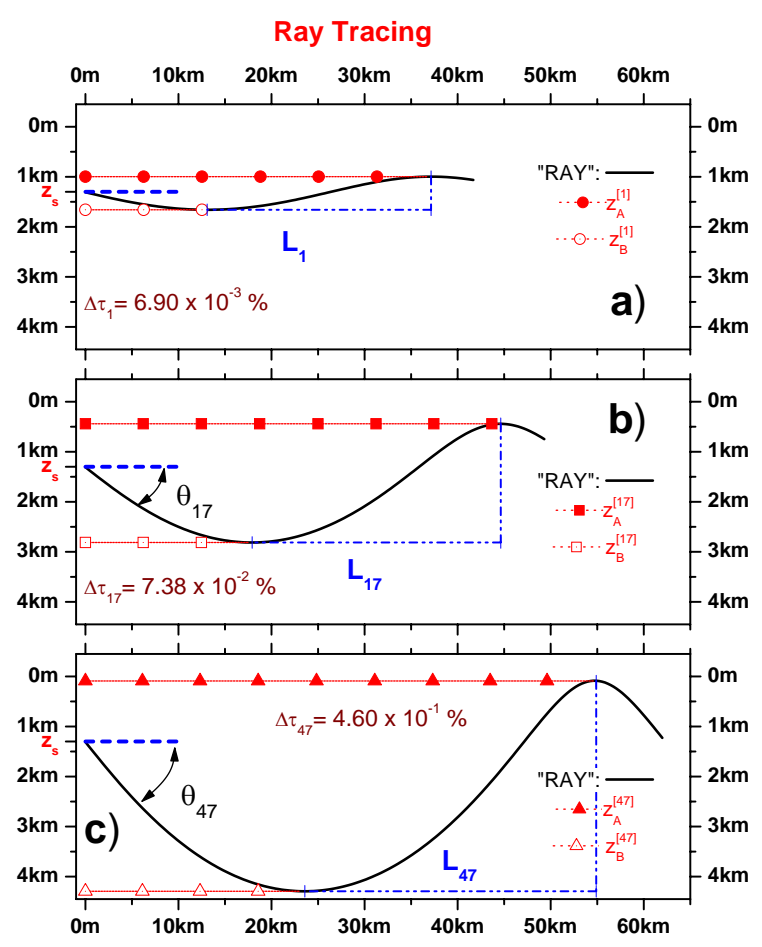

Figure 2 - Ray tracing for a $f=50 \mathrm{~Hz}$ source frequency, positioned in the SOFAR in $z_{s}=z_{\min }=1300 \mathrm{~m}$. In a), b) and c), the initial output angles of the source were the discrete modal angles $\theta_{n}$ with $n=1,17$ and 47 , respectively. It is noted in all these cases that the ray that passed through the turning points $z_{B}^{[n]}$ and $z_{A}^{[n]}$ after traveling radially the length $L_{n}$, the relative percentage difference $\Delta \tau_{n}$ between $\tau_{\Gamma}$ (Eq. 9 ) and $\tau_{n}$ (Eq. 10) is less than $0.5 \%$, showing an equivalence between modal and ray tracing theories in full accordance with Chapman \& Ellis (1983).

Therefore for each mode $n$, in the vicinity of the turning points $z_{A}^{[n]}$ and $z_{B}^{[n]}$, the wavefront moves with velocity similar to the sound velocity profile $c$.

On the other hand, with respect to the plane $z=z_{s}$ (parallel to the surface), where the source is located and wich contains $k_{r}^{[n]}$, we can make a complementary geometric view of modes (Figs. 3 and 2). Relating $k_{r}^{[n]}$ to the discrete shooting angles $\theta_{n}$, expressed by

$$
\theta_{n} \equiv \arccos \left(\frac{c_{\min }}{c_{p}^{[n]}}\right)
$$

With these ideas in mind, we will now attempt to analyze the problem of how should behave an Airy beam that has characteristics similar to propagating modes in a Munk acoustic guide.

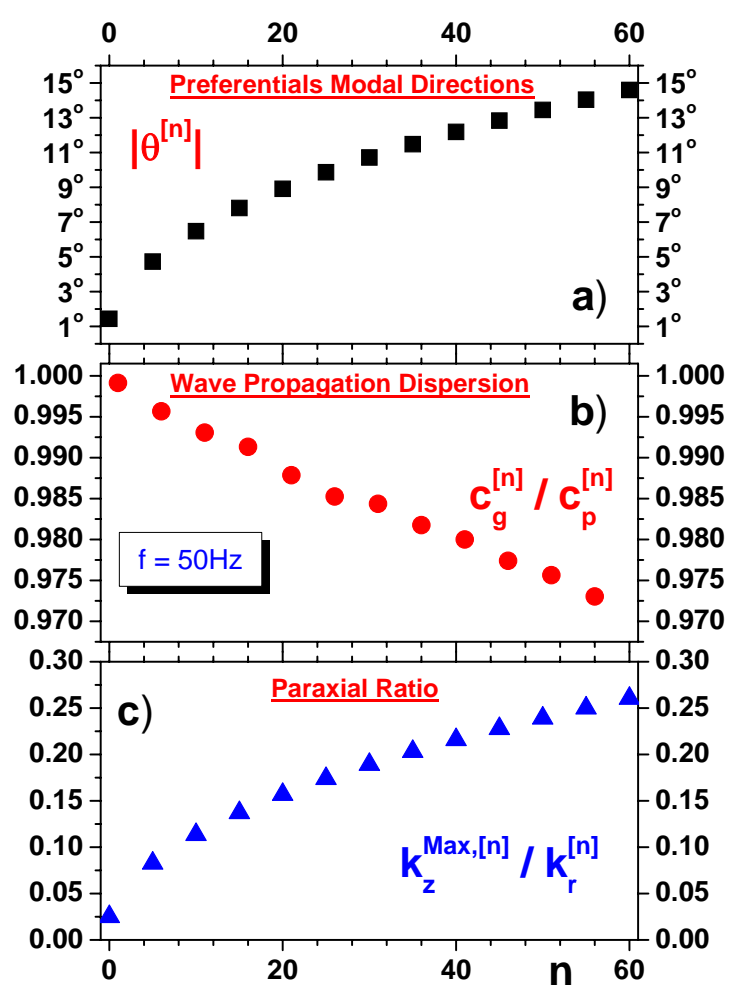

Figure 3 - Normal modes propagation parameters of the acoustic wave field, within the WKB formalism, to the Munk profile, with frequencies of $50 \mathrm{~Hz}$. The a) shows the behavior of the preferred angles of propagation in the range of $0<\left|\theta_{n}\right|<15^{\circ}$, associated with normal modes $n$ ranging from $0 \leq n \leq 60$. b) and c) tell us that these modes are not very dispersive, since $c_{g}^{[n]} \approx c_{p}^{[n]}$ and its propagation occurs mostly paraxially in the radial direction.

\section{RESULTS: AIRY DISCRETE BEAM PROPAGATION}

We have seen in the previous section that non-dispersive modes propagating paraxially (Chapman \& Ellis, 1983), may be associated with rays that are delimited between regions which are classically allowed and forbidden (Figs. 3 and 2). Motivated by these results, let's discuss the possibility of the existence in deep oceans of discrete Airy beams.

For a given mode $k_{r}^{[n]}$ associated with the Munk profile (Eq. 4), we propose that the discrete displacement field $\Phi_{n}(r, z)$, solution of the wave equation (Eq. 1), behave as

$$
\Phi_{n}(r, z) \equiv \frac{\left.\Psi_{n}(r, z) e^{i\left(k_{r}^{[n} r+k_{z}^{(n)}\left(z-z_{s}\right)\right.}\right)}{\sqrt[4]{r^{2}+\left(z-z_{s}\right)^{2}}} .
$$

We observed in Eq. (16) that, both the Sommerfeld radiation condition and the singular behavior of the Green function, in the vicinity of the source, are satisfied. 
On the other hand, assuming that $\Psi_{n}(r, z)$ varies more rapidly in depth than radially, the paraxial beams theory says that $\Psi_{n}$ asymptotically satisfies the following Schrödinger differential equation like,

$$
2 i k_{r}^{[n]} \frac{\partial}{\partial r} \Psi_{n}(r, z)+\frac{\partial^{2}}{\partial z^{2}} \Psi_{n}(r, z) \approx 0 .
$$

Since the 80's (Berry \& Balazs, 1979), the beams theory shows that Airy function may be solution of PDE's like Eq. (17). However, Airy functions are not square-integrable because, among others, it has a slow decay and has only one turning point. Therefore such beams do not preserve energy when they propagate into unlimited media. To overcome this strong constraint, for example, Airy beams are convolved with Gaussian beams (Bandres \& Gutiérrez-Vega, 2007).

However, here we are addressing the problem of Munk waveguide modes, and we will adopt a different strategy that consists of correlating two Airy beams in SOFAR; one associated to the region between the surface and SOFAR delimited by the depths $0 \leq z \leq z_{\min }$; and other associated to the region $z_{\min } \leq$ $z<\infty$, where the propagation occurs between SOFAR and the deep ocean.

Furthermore, in analogy with the problem of Schrödinger for quantum mechanics, we will require that the acoustic energy flow be preserved at $z=z_{\min }$, which means that the logarithmic derivative of the beam is preserved in $z=z_{\text {min }}$. Thus, in conformity with Berry \& Balazs (1979), we will require that the beam, when initiating the propagation in $r=0$, can be described by the following composition of Airy functions, related to $n$-nth mode of the Munk profile, namely

$\Psi_{\mathrm{n}}^{0}(\mathrm{z}) \equiv \Psi_{\mathrm{n}}(\mathrm{r}=0, \mathrm{z})=\mathrm{A}_{\mathrm{n}}\left\{\begin{array}{ll}A i\left(\frac{z_{A}^{[n]}-z}{\lambda_{n}}\right) & \text { if } 0 \leq z \text { and } z \leq z_{\min } \\ B_{n} A i\left(\frac{z-z_{B}^{[n]}}{\lambda_{n}}\right) & \text { if } z_{\min }<z\end{array}\right.$,

where $\operatorname{Ai}(u)$ is the Airy function of argument $u, A_{n}$ is an arbitrary normalization constant, and the turning points $z_{A}^{[n]}$ and $z_{B}^{[n]}$, associated with the Munk profile (Eq. 4), are calculated in the WKB approximation, discussed in the previous section (see Eq. 7). Moreover, as a consequence of the continuity of $\Psi_{n}^{0}$ and its derivative in $z=z_{\text {min }}$, we have that $B_{n}$ satisfies the equation

$$
B_{n}=\frac{A i\left(-\frac{z_{\min }-z_{A}^{(n)}}{\lambda_{n}}\right)}{A i\left(-\frac{z_{B}^{(n)}-z_{\min }}{\lambda_{n}}\right)}
$$

where, $\lambda_{n}$ is the wavelength of the Airy beam (see Figs. 4 and 5), and they are the $n$-nth solution of the transcendental equation (20), given by

$$
\begin{aligned}
& A i\left(\frac{z_{A}^{[n]}-z_{\min }}{\lambda_{n}}\right) A i^{\prime}\left(\frac{z_{\min }-z_{B}^{[n]}}{\lambda_{n}}\right)= \\
= & -A i\left(\frac{z_{\min }-z_{B}^{[n]}}{\lambda_{n}}\right) A i^{\prime}\left(\frac{z_{A}^{[n]}-z_{\min }}{\lambda_{n}}\right),
\end{aligned}
$$

where we denoted $A i^{\prime}(u)$ as the first derivative of of Airy function $A i(u)$, with respect to the argument $u$.

We observe in the Figure 5 that, for a given $n$, the amplitude of the initial Airy beam $\left(\left|\Psi_{n}^{0}\right|\right)$ has $(n+1)$ peak values spaced from $\lambda_{n}$, as the depth $z$ varies. In this sense and within the WKB approximation, we have that an estimate for $\lambda_{n}$ is given by

$$
\lambda_{\mathrm{n}} \approx\left(\frac{2\left[\sqrt{\left(\mathrm{z}_{\min }-\mathrm{z}_{\mathrm{A}}^{[\mathrm{n}]}\right)^{3}}+\sqrt{\left(\mathrm{z}_{\mathrm{B}}^{[\mathrm{n}]}-\mathrm{z}_{\min }\right)^{3}}\right]}{3 \pi(\mathrm{n}+1 / 2)}\right)^{2 / 3} .
$$

From Eq. (21) and Figures 5 and 6, we see that, as $n$ increases the value of $\lambda_{n}$ decreases.

Moreover, it is noted in both Figures 4 and 5 , that in the vicinity of the turning points, $z_{A}^{[n]}$ and $z_{B}^{[n]}$, the beam $\Psi_{n}^{0}$ reaches its maximum amplitude before evanescing.

Again using the WKB method, we can obtain an estimate of how much the beam penetrates the region of penumbra, until it falls to a value of around $30 \%$ of its maximum amplitude. For this, we denote $\sigma_{n}$ as the penetration length, namely

$$
\sigma_{n} \approx \lambda_{n}\left(\frac{3}{2}\right)^{2 / 3}\left[1+\frac{(2 \pi)^{2 / 3}}{2}\right] \approx 3.54 \lambda_{n} .
$$

It means that the acoustic energy (see Figs. 5 and 6 ) penetrates in the region of penumbra, significantly, a greater distance than triple wavelength $\lambda_{n}$.

Finally, similar to the theory developed in Berry \& Balazs (1979), it is shown that for the initial condition $\Psi_{n}^{0}$ (Eq. 18), the envelope $\Phi_{n}$ of the field $\Phi_{n}$ (Eq. 16) satisfies the following equation

$$
\begin{aligned}
& \Psi_{n}(r, z)= \\
& =A_{n} \begin{cases}A i\left(-\left[z+r^{2}\left(4 z_{\min } k_{r}^{[n]}{ }^{2} \lambda_{n}^{3}\right)^{-1}-z_{A}^{[n]}\right] \lambda_{n}^{-1}\right) & \text { if } 0 \leq z \leq z_{\min } \\
B_{n} A i\left(\left[z-r^{2}\left(4 z_{\min } k_{r}^{[n)^{2}} \lambda_{n}^{3}\right)^{-1}-z_{B}^{[n]}\right] \lambda_{n}^{-1}\right) & \text { if } z_{\min }<z\end{cases}
\end{aligned}
$$

On the other hand, we have seen in previous sections that beyond the extreme sensitivity of the beam to the initial condition 


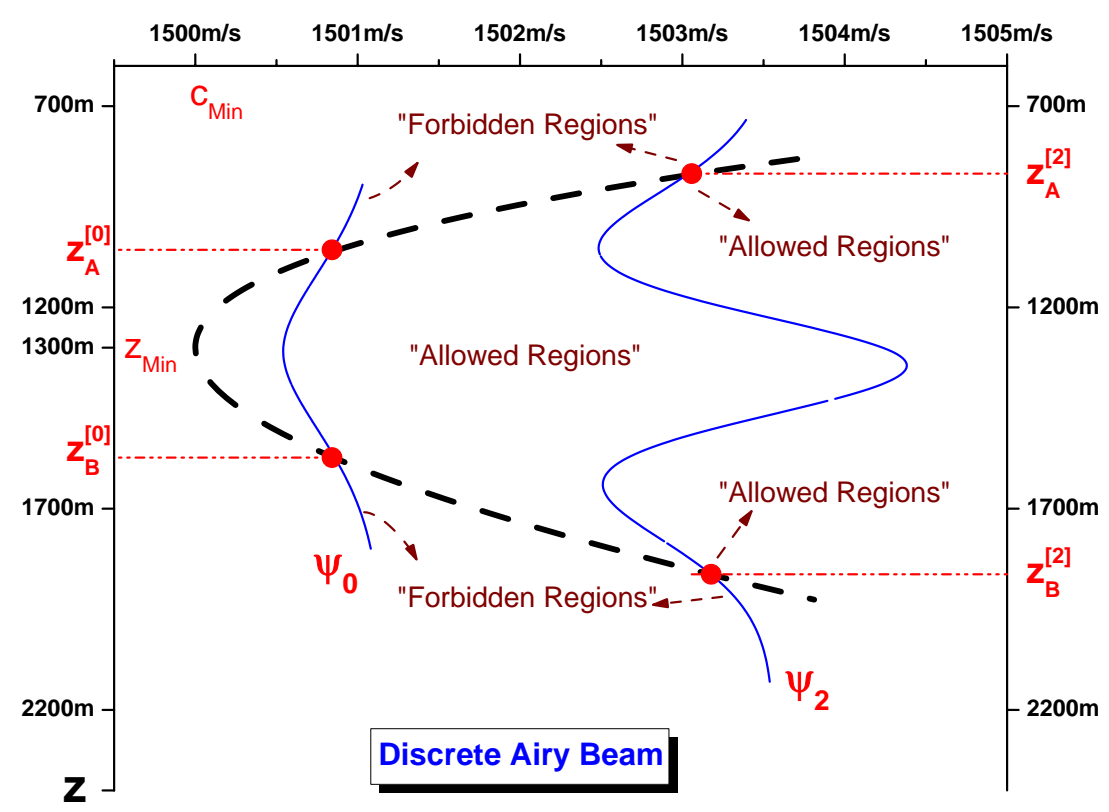

Figure 4 - The behavior of initial condition $\Psi_{n}^{0}$ for the beams, with $f=50 \mathrm{~Hz}$ and $r=0$ (see Eq. 18). Note that for any $n$-discrete, $\Psi_{n}^{0}$ is evanescent in the classically forbidden regions and oscillates $(n+1)$-times in the classically allowed regions.

$\Psi_{n}^{0}$ in Eq. (18), $\Phi_{n}$ in Eq. (16) has a behavior that is intended to propagated in classically allowed or forbidden regions.

In the first region the beam can oscillate enough, while in the other it is always evanescent. To bypass these difficulties inherent for this type of beam, we will use the strategy of associating each mode $n$, of Munk profile, with a trajectory $\Gamma \mapsto \Gamma_{n}$ of a ray, with angle of shot $\theta_{n}$, and throughout its propagation, it has traveled radially, that at least, half length of modal interference $L_{n}$ (see Fig. 2).

With this, we hope to guarantee a lower dispersion, as well as to maintain the validity of the paraxial approximation of the beam (see Fig. 3).

In this sense, the computation of their transmission losses $T L_{\Gamma_{n}}$ (Jensen, 1994), provides us a good quantitative picture on the physical behavior of the beam $\Phi_{n}$ (Eq. 16) as its propagates over $\Gamma_{n}$, which is given by

$$
T L_{\Gamma_{n}} \equiv 20 \log _{10}\left(\left|\frac{\Phi_{n}\left(r\left(\Gamma_{n}\right), z\left(\Gamma_{n}\right)\right)}{\Phi_{n}^{s}}\right|\right) .
$$

where $\Phi_{n}^{s}$ is the reference value of the beam, to the equivalent at irradiated pressure of $1 \mu \mathrm{Pa}$ at the distance of $r=1 \mathrm{~m}$, beyond the position of the source in $z=z_{s}$ (Jensen, 1994).

Therefore, within the WKB approximation to characterize the allowed and forbidden areas of the Munk waveguide for frequency of $50 \mathrm{~Hz}$, first we trace the ray with shooting angles $\theta_{n}$, which trajectories $\Gamma_{n}$ traveled, at least, the radial distance of $L_{n}$ (see Fig. 7a).

Subsequently, we calculated the values of $T L_{\Gamma_{n}}$ (Eq. 24) in the points of the trajectory $\Gamma_{n}$ (see Fig. 7b). As the wavelengths $\lambda_{n}$ and penetration $\sigma_{n}$ change with $n$ (see Fig. 6), several regions of the Munk waveguide can be sounded, not just those classically allowed, but also large extensions of the forbidden areas.

\section{CONCLUSIONS, FINAL REMARKS AND FURTHER PERSPECTIVES}

In this work, based on the pioneering ideas of Berry \& Balazs (1979) and Berry (2005), we have developed a model that shows that it is possible to propagate Airy beams over long distances and to sonify vast regions of a deep ocean (see Fig. 7b). In order to do so, we first had to adapt these beams to the fundamental characteristics of the propagating modes of Munk's acoustic waveguide (Eq. 4).

More explicitly, within the WKB formalism (Eq. 5) we calculate the discrete radial wave numbers $k_{r}^{[n]}$ and the respective turning points $\left(z_{A}^{[n]}\right.$ and $\left.z_{B}^{[n]}\right)$ associated with a given mode $n$ of the Munk waveguide (see Figs. 4 and 5). Then, to use the equivalence between the normal modes and ray theories (Chapman \& Ellis, 


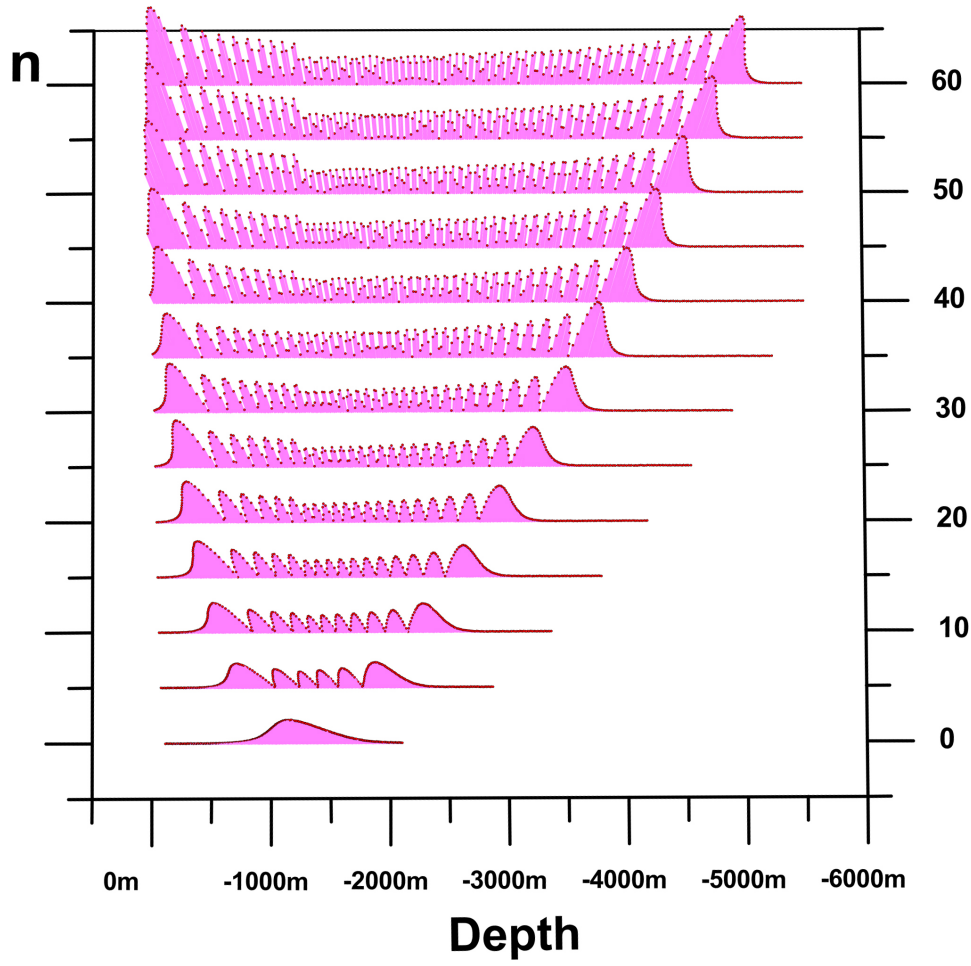

Figure 5 - For $r=0$ and $f=50 \mathrm{~Hz}$, in arbitrary units and in function of the depth, shows the behavior of the amplitude $\left|\Psi_{n}^{0}\right|$ of initial condition for the discrete Airy beam (see Eq. 18). Note that as $n$ increases, there are $(n+1)$ peaks equally spaced in $\lambda_{n}$ (see Eq. 21). It is also noted that the highest intensity peaks occur in the neighborhoods of the turning points $z_{A}^{[n]}$ and $z_{B}^{[n]}$ (see Fig. 4) and the beam penetrates beyond them, evanescently (see Eq. 22).

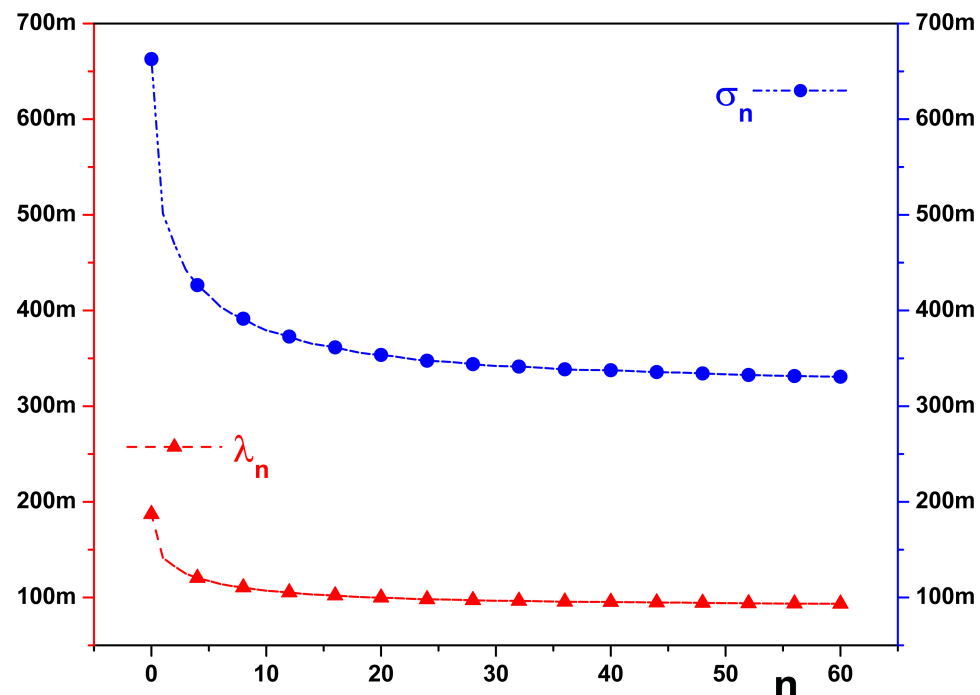

Figure $\mathbf{6}$ - Comparation of the wavelengths $\lambda_{n}$ and penetration lengths $\sigma_{n}$ as a function of $n$, for $f=50 \mathrm{~Hz}$. Note that the higher values of both are reached for the smallest values of $n$, and both $\lambda_{n}$ and $\sigma_{n}$ decrease more slowly when $n$ increases. It is also noted that the values of $\sigma_{n}$ are always higher than $\lambda_{n}$ (see Eqs. 21 and 22). 


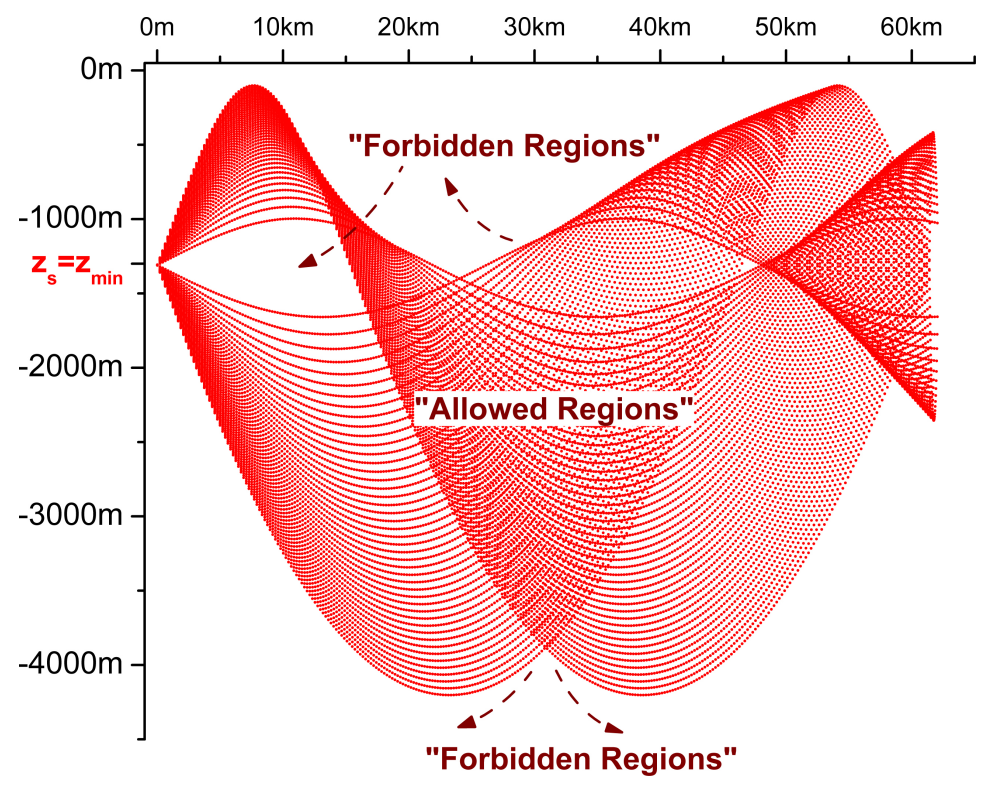

a) Ray Tracing "Grid"

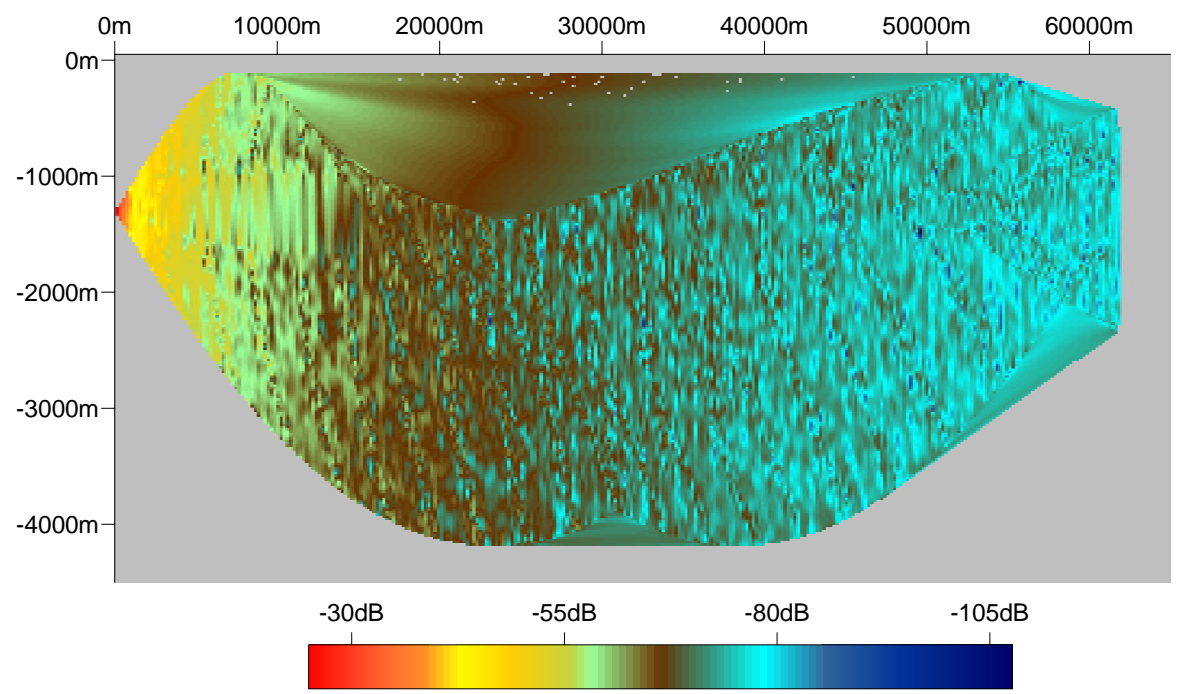

b) Discrete Airy Beams Transmission Loss (dB)

Figure 7 - Wave field modeling for Munk profile (Eq. 4) and $f=50 \mathrm{~Hz}$. a) Ray tracing with all the possible trajectories $\Gamma_{n}$ of the acoustic rays associated with a discrete set of shooting angles $\theta_{n}$, with $0<\left|\theta_{n}\right|<15^{\circ}$ and $0 \leq n \leq 60$. Note that such trajectories $\Gamma_{n}$ delimit the regions classically allowed and forbidden. b) Airy beam propagation $\Phi_{n}$ showing the transmission losses $T L_{\Gamma_{n}}$ (Eq. 24) in a color map (dB) Note that for a discrete Airy beam trajectory $\Gamma_{n}$, the acoustic energy propagates in regions that are classically permitted as well as substantially penetrate into the forbidden ones. 
1983), we associate each wave vector $k_{r}^{[n]}$ (normal mode theory) with shooting angles $\theta_{n}$ (ray theory), from a source positioned at $z=z_{s}$ (see Figs. 3, 4 and 5).

Finally, as shown in Fig. 7b, we analyze the propagation of discrete Airy beams (see Eqs. 16 and 23) along of $n$ rays trajectories $\Gamma_{n}$. We verified that the acoustic energy propagates in the classically allowed regions and has penetrated substantially in the classically forbidden regions (see Fig. 7b).

Despite of its computational cost to be a little high, since the calculation of the discrete Airy beam, in Eq. (24), requires, previously, the use of some numerical methods, in extended precision, for the development of WKB theory and the subsequent ray tracing, we believe that present theory can improve accuracy in some inversion problems in oceanographic acoustic tomography (Marin et al., 2016).

We show here that discrete Airy beams carry acoustic energy in both classically allowed and forbidden regions with modal times equivalent to travel times (see Fig. 2). We can use this fact to adapt the usual algorithms of travel time tomography (Popov, 2002; Bleistein, 2008; Marin et al., 2016) to the present theory of discrete Airy beams. Efforts in this direction are being made and planned to published shortly.

\section{ACKNOWLEDGMENTS}

The authors would like to thanks Prof. Carlos Eduardo Parente Ribeiro for many discussions and helpful suggestions given during the development of this work.

\section{REFERENCES}

BANDRES MA \& GUTIÉRREZ-VEGA JC. 2007. Airy-Gauss beams and their transformation by paraxial optical systems. Optics Express, 15(25): 16719-16728.
BERRY MV. 2005. Tsunami asymptotics. New Journal of Physics, 7(1): $1-18$.

BERRY MV \& BALAZS NL. 1979. Nonspreading wave packets. Am. J. Phys, 47(3): 264-267.

BLEISTEIN N. 2008. Mathematics of modeling, migration and inversion with Gaussian beams. In: 70th EAGE Conference and Exhibition-Workshops and Fieldtrips, p. 7-36. Rome, Italy: EAGE.

CERVENY V. 2005. Seismic ray theory. New York: Cambridge University Press. 724 pp.

CHAPMAN DMF \& ELLIS DD. 1983. The group velocity of normal modes. The Journal of the Acoustical Society of America, 74(3): 973-979.

DURNIN J, MICELI Jr JJ \& EBERLY JH. 1987. Diffraction-free beams. Physical Review Letters, 58(15): 1499-1501.

FU S, ZHOU J, LI Y, SHEMER L \& ARIE A. 2017. Dispersion Management of Propagating Waveguide Modes on the Water Surface. Physical Review Letters, 118(14): 144501.

JENSEN FB. 1994. Computational ocean acoustics. Springer Science \& Business Media. 794 pp.

MARIN FO, RODRÍGUEZ OC, GUIMARÃES LG \& RIBEIRO CEP. 2016. Modal inversion by using parameterization with Chebyshev polynomials. Brazilian Journal of Geophysics, 34(3): 385-392.

POPOV MM. 2002. Ray theory and Gaussian beam method for geophysicists. Salvador, BA, Brazil: Edufba. 172 pp.

PORTER MB \& BUCKER HP. 1987. Gaussian beam tracing for computing ocean acoustic fields. J. Acoust. Soc. Am., 82(4): 1349-1359.

SALANDRINO A \& CHRISTODOULIDES D. 2011. Airy plasmons defeat diffraction on the surface. Physics, 4: $69 \mathrm{pp}$.

SIVILOGLOU GA \& CHRISTODOULIDES DN. 2007. Accelerating finite energy Airy beams. Optics Letters, 32(8): 979-981. 